\title{
A systematic study of biochemical profile during the induction and development of an animal model for Metabolic Syndrome
}

\author{
D'Souza Serena Stephen and Asha Abraham* \\ Fr George Albuquerque Pai Cell and Molecular Biology Laboratory. \\ Department of Post Graduate Studies and Research in Biotechnology, St Aloysius College, Mangalore- 575003
}

\begin{abstract}
Metabolic syndrome is a multiplex risk factor arising from abdominal obesity, insulin resistance and dyslipidemia. It increases the risk for cardiovascular complications, fatty liver disease and cognitive dysfunction. The prevalence is rampantly increasing among all age groups worldwide due to nutrition excess, physical inactivity and increased stress. Despite its epidemiological nature, treatment is limited to lifestyle changes and lifelong medical intervention. This may be due to incomplete knowledge of the etiology of the syndrome. The present study is aimed at developing an animal model using male C57BL/6J mice and studying the biochemical profile during the onset and progression of the syndrome. Test group was fed with High Fat Simple Carbohydrate (HFSC) diet and the Control group was fed with standard diet for a period of 5 months. Body weight and biochemical profile was analyzed on a monthly basis. Oral Glucose tolerance test (OGTT) was performed following a glucose load of $1 \mathrm{~g} / \mathrm{kg}$ body weight at $143^{\text {rd }}$ day. The syndrome was successfully induced in male C57BL/6J mice within a period of 5 months with perturbed lipid profile and insulin resistance.
\end{abstract}

Keywords: C57BL/6J, Metabolic Syndrome, High Fat Simple Carbohydrate (HFSC) Diet

\section{Introduction}

Metabolic Syndrome, a cluster of lipid abnormalities and insulin resistance, is increasing at an epidemiological rate worldwide. The risk factors that aggregate to cause the syndrome include High blood pressure, High Triglycerides, Low High Density Lipoprotein, High Low Density Lipoprotein and High Fasting Blood Glucose [1]. The metabolic syndrome has important health implications as it precedes cardiovascular diseases, cognitive problems, delayed blood clotting, inflammatory disorders etc. The syndrome was thought to be prevalent only in adults. However, studies show that it is rampant in all age groups. $29.2 \%$ of obese children are found to be inflicted with the syndrome with a higher prevalence in boys than girls [2]. The prevalence of the MetS among hypertensive population was $31.2 \%$. The sex-specific prevalence's were $15.1 \%$ and $47.6 \%$ among male and female patients respectively. A large number of the patients $(40.4 \%)$ were at a high potential risk of developing the MetS as they already met 2 of the criteria [3].

Stress, physical inactivity and nutrition excess are considered to be the prime causative factors. Nutrition excess mainly concerns which food component is consumed. Consumption of frequent unbalanced diets such as those rich in fat such as animal fat and carbohydrate particularly simple carbohydrates accelerate the path to obesity. The treatment regimen includes lifestyle changes such as weight control, exercise, improved nutrition and lifelong medical intervention. Besides morbidity and mortality, these disorders impact the socioeconomic status of individuals as well as state [4].Evaluation of the etiology of the syndrome will be imperative in finding a possible effective cure. Most of the studies carried out in this area include clinical trials which are limited to prevalence studies [5],[6],[7].Therefore in our study, we have aimed at developing an animal model which is induced with metabolic syndrome which will help in carrying out in depth studies on the syndrome.

\subsection{Animals used:}

\section{Materials}

Male C57BL/6J mice were used during this study. They were maintained at $25 \pm 2{ }^{\circ} \mathrm{C}$, twelve hour light and dark cycle and fed with lab chow and water ad libtium according to the CPCSEA guidelines with approval from Institutional animal ethics committee (Sanction No. SAC/IAEC/110/2011 dated $30^{\text {th }}$ March, 2011).

\subsection{Chemicals used:}

2.2.1. Feed used: Control and Test feed was prepared from local ingredients

2.2.2. Kits used: Blood Glucose, Blood Cholesterol, Blood Triglycerides, High Density Lipoprotein and Low Density Lipoprotein were analyzed using Agappe kits (Kerala, India)

2.2.3. Other chemicals: All chemicals used were of analytical grade obtained from Himedia, Blood glucose during Oral Glucose Tolerance Test (OGTT) was analyzed using Glucometer 


\subsection{Induction of Metabolic Syndrome}

\section{Methods}

Pathogen free male C57BL/6J mice were used in this study. Four week old male C57BL/6J mice were divided into Control and Test groups $(\mathrm{n}=6$ ). The test group was fed with High Fat Simple Carbohydrate (HFSC) Diet and the control group was fed with Standard diet for a period of five months. The HFSC Diet comprised of 46\% Fat, $42 \%$ Simple Carbohydrate and $12 \%$ Protein. The food was changed daily. Anthropometric parameters such as body weight and Body Mass Index were monitored on a monthly basis.

\subsection{Ascertaining induction of the Metabolic Syndrome}

\subsubsection{Biochemical analysis}

The animals were bled at 0 day, 1 month, 2 month, 3 month, 4 month and 5 month of feeding by tail vein bleeding. The blood was collected by tail vein bleeding and centrifuged at $2000 \mathrm{~g}$ for 10 minutes at room temperature to obtain serum. Blood Triglycerides, Blood Cholesterol, Blood Glucose was monitored on a monthly basis. High Density Lipoprotein (HDL) and Low Density Lipoprotein (LDL) were measured after 5 months of feeding. All analysis was carried out using kits (Agappe, India).

\subsubsection{Oral Glucose Tolerance Test [8]}

The glucose tolerance was tested after 4 months and 3 weeks of feeding by performing Oral Glucose Tolerance test (OGTT).The animals were starved for $18 \mathrm{~h}$ prior to a glucose load at rate of $1 \mathrm{~g}$ per kg body weight and was administered orally at the rate of $1 \mathrm{~g} / \mathrm{ml}$ solution .Blood glucose was tested at 0, 30, 60, 90 and $120 \mathrm{~min}$ using a glucometer.

\subsubsection{Insulin analysis}

The insulin analysis was carried out using serum obtained during Oral Glucose Tolerance Test (OGTT). The insulin was analyzed at $0 \mathrm{~min}, 30 \mathrm{~min}$ and $120 \mathrm{~min}$ of glucose load using Ultrasensitive Mouse Insulin ELISA Kit, Crystal Chem. Inc., USA

\section{Figures And Tables}

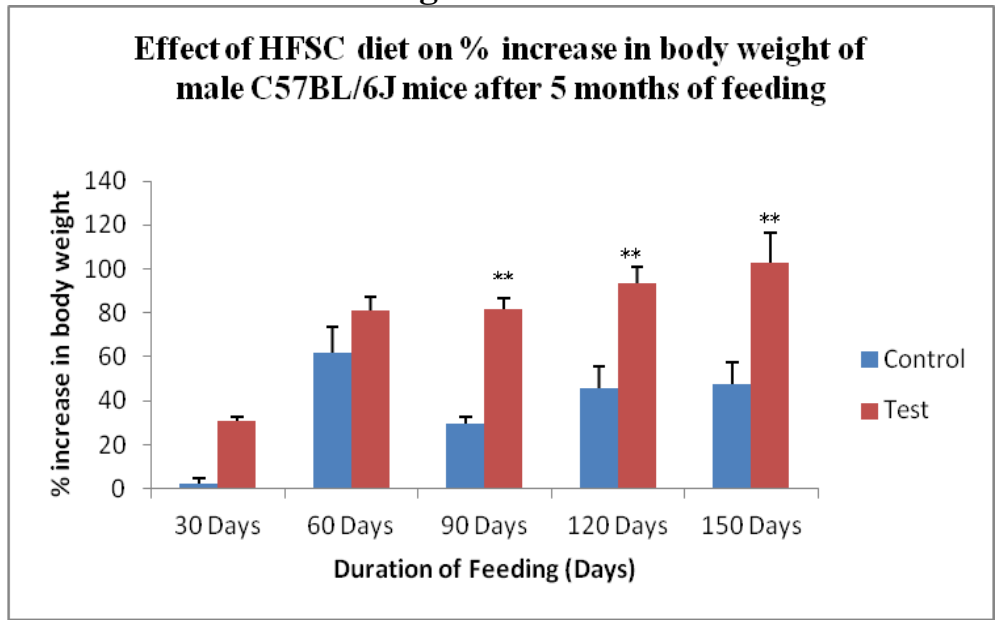

Figure 1: Effect of HFSC Diet on \% increase in body weight of male C57BL/6J mice after 5 months of feeding

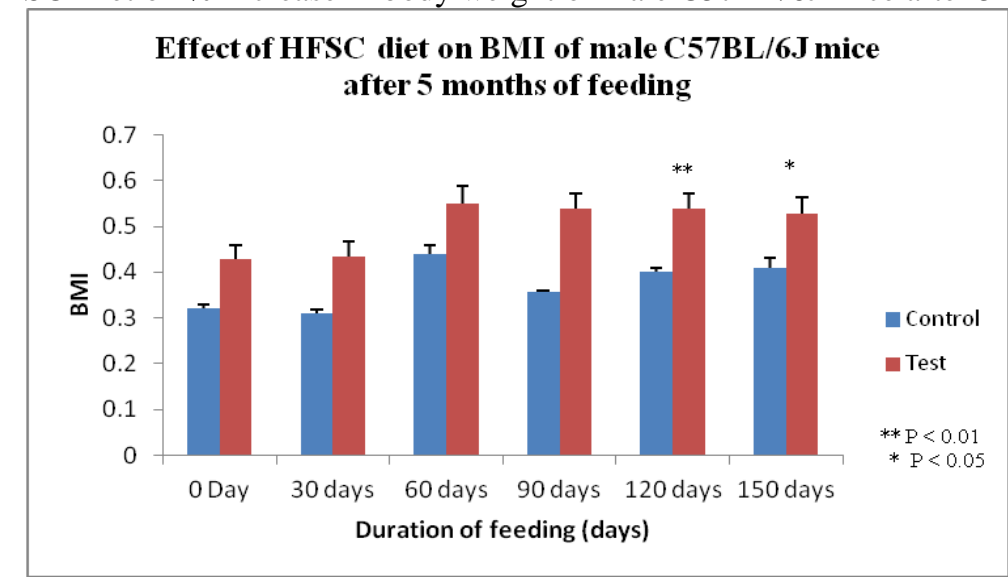

Figure 2: Effect of HFSC Diet on Body Mass Index (BMI) of male C57BL/6J mice after 5 months of feeding 


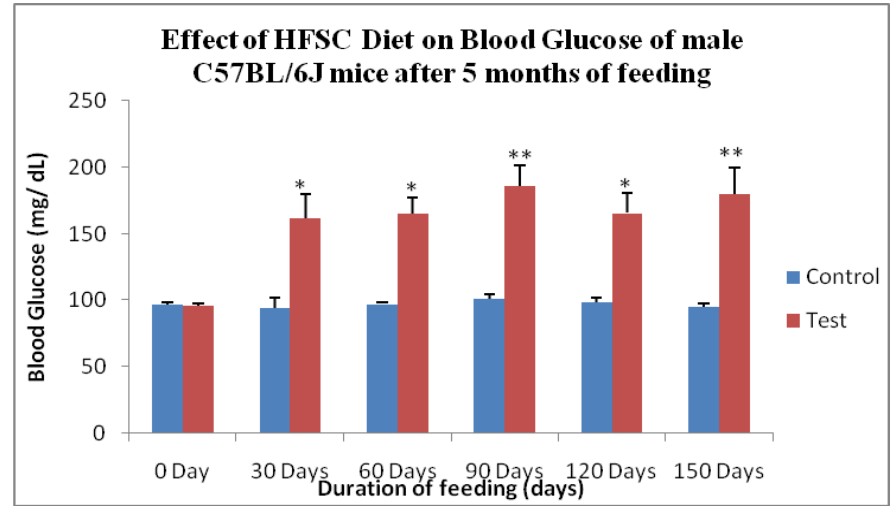

Figure 3: Effect of HFSC Diet on Blood Glucose of male C57BL/6J mice after 5 months of feeding

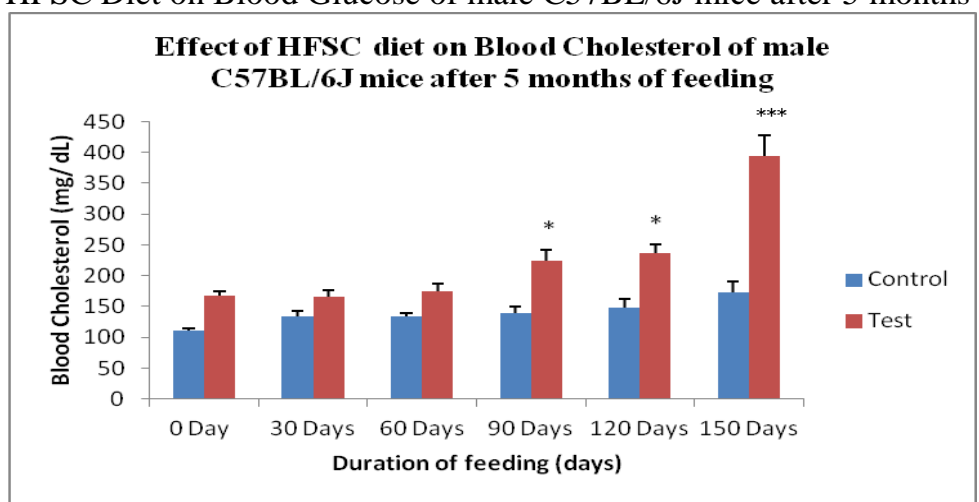

Figure 4: Effect of HFSC Diet on Blood Cholesterol of male C57BL/6J mice after 5 months of feeding

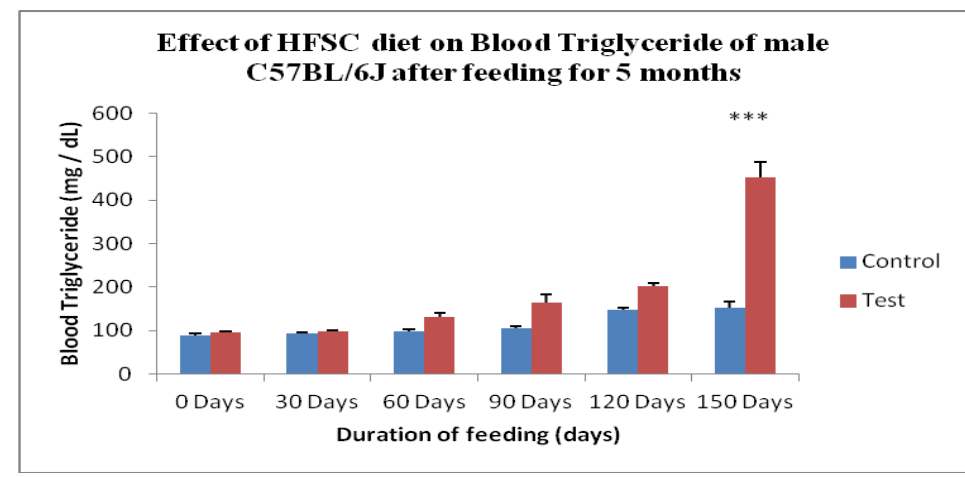

Figure 5: Effect of HFSC Diet on Blood Triglycerides of male C57BL/6J mice after 5 months of feeding

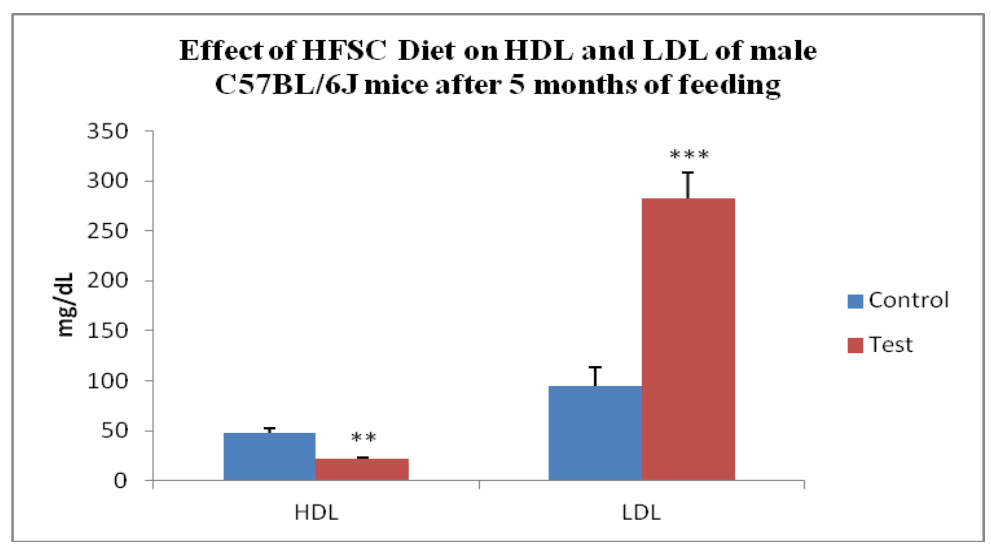

Figure 6: Effect of HFSC Diet on High Density Lipoprotein (HDL) and Low Density Lipoprotein (LDL) of male C57BL/6J mice after 5 months of feeding 


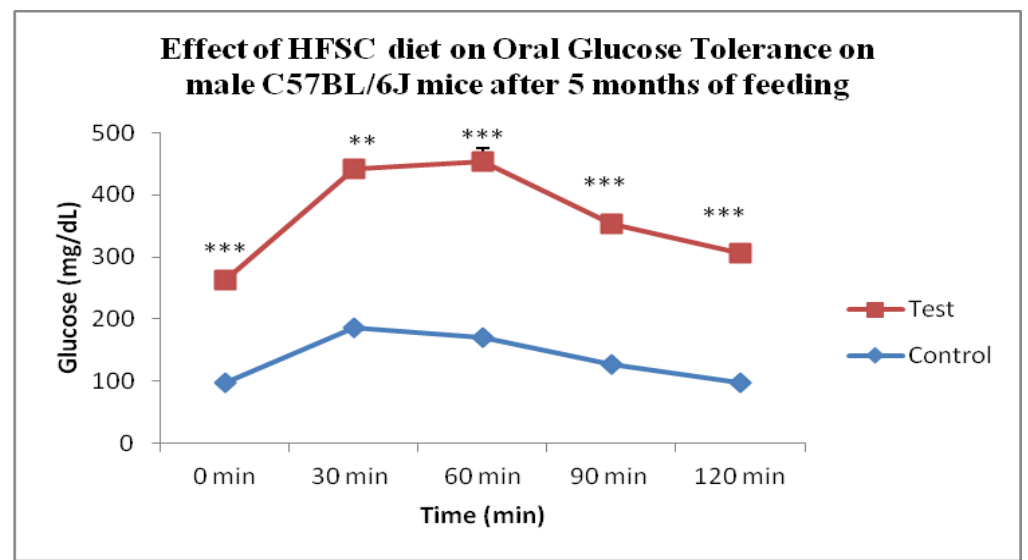

Figure 7: Effect of HFSC Diet on Oral Glucose Tolerance of male C57BL/6J mice after 5 months of feeding

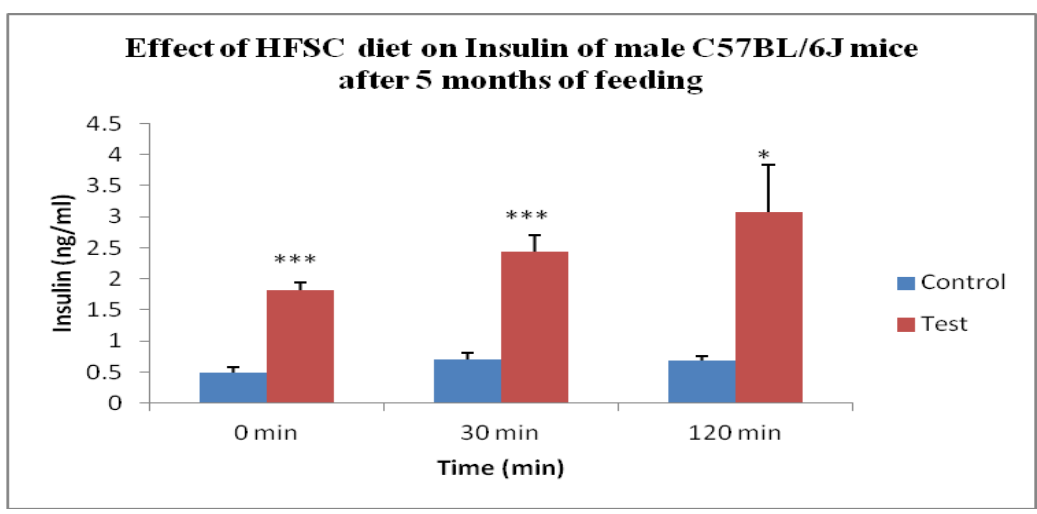

Figure 8: Effect of HFSC Diet on Oral Glucose Tolerance of male C57BL/6J mice after 5 months of feeding

\section{Results And Discussion}

Metabolic syndrome was induced by feeding a formulated High Fat Simple Carbohydrate (HFSC) diet in with modifications [9], to one month old male C57BL/6J mice for 5 months. The induction was ascertained by analyzing lipid profile and insulin resistance.

A significant increase in the body weight of the test was observed at 90, 120 and $150(\mathrm{p}<0.01)$ days respectively as compared to the control (Fig. 1). A significant increase in test Body Mass Index (BMI) was observed after 120 days $(\mathrm{p}<0.01)$ of feeding and was maintained till 150 days $(\mathrm{p}<0.05)$ as compared to Control (Fig. 2). Blood Glucose levels of the test increased significantly after 30 days $(p<0.05)$ of feeding and was maintained till 150 days $(\mathrm{p}<0.01)$ (Fig. 3).

The test cholesterol increased after 90, 120 days ( $p<0.05)$ till 150 days of feeding ( $p<0.001)$ (Fig. 4). However, triglyceride levels in the test group were also significantly higher after 120 days of feeding $(\mathrm{p}<0.001)$ (Fig.5). The test group was also seen to have decreased HDL levels $(p<0.01)$ and increased LDL levels $(\mathrm{p}<0.001)$ as compared to control post 5 months of feeding (Fig.6). This explains elevated Cholesterol levels as low HDL levels retard reverse cholesterol transport [10]. Elevated blood LDL may be due to increased fat load in the liver which causes the liver to produce excessive lipoproteins triacylglycerol [11].

The test group also demonstrated impaired glucose tolerance with a significant increase in blood glucose at 0 ( $<<0.001), 30(\mathrm{p}<0.01), 60(\mathrm{p}<0.001), 90(\mathrm{p}<0.001)$ and $120(\mathrm{p}<0.001)$ minutes respectively as compared to the control post glucose load (Fig. 7). A corresponding significant increase was also observed in the test insulin levels at $0(\mathrm{p}<0.001), 30(\mathrm{p}<0.001)$ and $120(\mathrm{p}<0.05) \mathrm{min}($ Fig. 8). This falls in line with the observed high Triglycerides. Insulin resistance is known to impair the storage of fatty acids by increasing the rate of fatty acid release by adipose tissue leading to the uptake of fatty acids to non adipose storage tissues [12].Increased Triglycerides, LDL, decreased HDL levels, impaired Oral glucose tolerance were observed in eight week old C57BL/6J mice fed with High Fat Diet for 16 weeks in earlier studies [9].

\section{Conclusion}

Metabolic syndrome was successfully induced in male C57BL/6J mice by feeding High Fat Simple Carbohydrate (HFSC) Diet for a period of five months. The syndrome was ascertained by analyzing 
biochemical parameters to obtain an animal model .This will enable further valuable research on the molecular aspects of metabolic syndrome

\section{Acknowledgement}

Dsouza Serena thanks CSIR for supporting this work with a Senior research fellowship. Dr. Asha Abraham thanks UGC and BRNS for supporting this work with a Major Research Project. All authors thank The Principal of St Aloysius College for providing the facilities and support to carry out this work. Also big thanks to Rev. Fr Leo Dsouza SJ (Director, Laboratory of Applied Biology) and his lab members and Miss Sushma Patrao, Lecturer, St Aloysius College for their valuable assistance.

\section{References}

[1] K. G. M. M. Alberti, P. Zimmet and J. Shaw. Metabolic syndrome-a new world-wide definition. A Consensus Statement from the International Diabetes Federation, Diabetic Medicine, 2006, 23, 469-48.

[2] F. Amanda, C. Leone, and T. Steve. The Prevalence of Metabolic Syndrome in Children:A Systematic Review of the Literature, Metabolic Syndrome And Related Disorders, 2013,11(2), 71-80.

[3] U. O. Charles and G. O. Emeka. Prevalence and characteristics of the metabolic syndrome among newly diagnosed hypertensive patients, Indian Journal of Endocrinology and Metabolism , 2012 ,16(1),S104-109.

[4] D. Ankhush and T. Nikhil. Challenges in prevention and management of diabetes mellitus and metabolic syndrome in India, Current Science, 2009; 97: 356-366.

[5] K. Shiwaku , A. Nogi, K. Kitajima, E. Anuurad, B. Enkhmaa, M. Yamasaki, J.-M. Kim, I.-S. Kim, S.- K. Lee, T. Oyunsuren and Y. Yamane. Prevalence of the Metabolic Syndrome using the modified ATP III definitions for workers in Japan , Korea and Mongolia, Journal of Occupational Health, 2005,47,126-135.

[6] R.M. Mabry, M.M. Reeves and E.G. Eakin, N. Owen. Gender differences in prevalence of the metabolic syndrome in gulf cooperation council countries: a systematic review, Diabetic Medicine: A journal of the British Diabetic Association, 2010, 27(5),593-597.

[7] Z. H. Nejhad, H. M. Vardanjani, F. A., M. Hadipour and K. Sheikhzadeh. Relative effect of socio-economic status on the healthrelated quality of life in type 2 diabetic patients in Iran, Diabetes \& Metabolic Syndrome: Clinical Research \& Reviews, 2013,7(4),187-190.

[8] R. Buettner, K.G. Parhofer, M. Woenckhaus, C.E. Wrede, L.A. Kunz-Schughart, J. Schölmerich and L.C. Bollheimer. Defining high-fat-diet rat models: metabolic and molecular effects of different fat types, Journal of Molecular Endocrinology, 2006; (36): $485-501$.

[9] J.C. Fraulob, R. Ogg-Diamantino, C. Fernandes-Santos, M. B. Aguila, and C. A. Mandarim-de-Lacerda A mouse model of Metabolic syndrome: Insulin resistance, fatty liver and non alcoholic fatty pancreas disease in C57/BL6 mice fed a high fat diet, Journal of Clinical Biochemistry Nutritions, 2010;(46):212-223.

[10] J. Savel, M. Lafitte, Y. Pucheu, V. Pradeau, A. Tabarin and T. Couffinhal. Very low levels of HDL cholesterol and atherosclerosis, a variable relationship - a review of LCAT deficiency, Vascular Health and Risk Management,2012,8, 357 - 361.

[11] K. L. Donnelly, C. I. Smith, J. Sarah, J. Jessurun, M. D. Boldt and E. J. Parks Schwarzenberg. Sources of fatty acids stored in liver and secreted via lipoproteins in patients with nonalcoholic fatty liver disease, The Journal of Clinical Investigation ,2005, 115 (5), 1143-1151

[12] A.J. Sanyal, C. Campbell-Sargent, F. Mirshahi, W.B. Rizzo, M.J. Contos, R.K. Sterling, V.A. Luketic, M.L. Shiffman and J.N. Clore. Nonalcoholic steatohepatitis: association of insulin resistance and mitochondrial abnormalities, Gastroenterology, 2001, 120(5), 1183-1192. 